\title{
ИСПОЛЬЗОВАНИЕ ТЕОРИИ НЕЧЕТКИХ МНОЖЕСТВ В ЭКОНОМИЧЕСКОМ АНАЛИЗЕ ИНВЕСТИЦИОННЫХ ПРОЕКТОВ
}

\author{
${ }^{1}$ Файзиев Р. А. к. физ. - мат. н., \\ ${ }^{l}$ Хаитматов У. Т. к. т. н., \\ ${ }^{\prime}$ Азаматов О. Х. К. э. н., \\ ${ }^{1}$ Джнуманиязов Ш. Р., \\ ${ }^{2}$ Хасанова $X . X$.

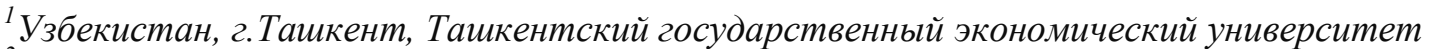 \\ ${ }^{2}$ Узбекистан, Ташкентская область, Алмаликский филиал Ташкентского технического \\ университета имени Ислама Каримова
}

DOI: https://doi.org/10.31435/rsglobal_ijite/01062018/5672

\section{ARTICLE INFO}

Received 18 April 2018

Accepted 21 May 2018

Published 01 June 2018

\section{KEYWORDS}

investment,

estimation,

efficiency,

uncertainty,

fuzzy,

logic,

set,

project,

factor,

mathematical apparatus

(C) 2018 The Authors.

\begin{abstract}
The article outlines the main features of the use of the theory of indefinite bundles in the evaluation of the cost-effectiveness of investment projects. $\mathrm{He}$ analysis of methods for quantifying the effectiveness of the IP under uncertainty suggests that the existing methods either eliminate the uncertainty from the IP model, which is inappropriate, since uncertainty is an integral characteristic of any forecast, or are unable to formally describe, and take into account all possible varieties of types of uncertainty.

Methods based on the theory of fuzzy sets refer to the methods of evaluation and decision-making under conditions of uncertainty. Their use implies the formalization of the initial parameters and performance targets of the IP in the form of a vector of interval values (fuzzy interval), the hit in each interval of which is characterized by a certain degree of uncertainty.

Also, the fuzzy-interval approach has advantages in solving the problems of forming an optimal portfolio of investment projects. To solve the problem of forming an optimal IP portfolio, a large number of models for the formation of an optimal IP portfolio have been developed, differing from each other in the form of objective functions, variable properties, used by mathematical methods, and uncertainty.
\end{abstract}

Введение. Как известно, процесс инвестирования играет важную роль в экономике любой страны. Инвестирование в значительной степени определяет экономический рост государства, занятость населения и составляет существенный элемент базы, на которой основывается экономическое развитие общества. Поэтому проблема, связанная с эффективным осуществлением инвестирования, заслуживает серьезного внимания.

Инвестиционная деятельность представляет собой один из наиболее важных аспектов функционирования любой коммерческой организации. Причинами, обусловливающими необходимость инвестиций, являются обновление имеющейся материально-технической базы, наращивание объемов производства, освоение новых видов деятельности.

Значение анализа для планирования и осуществления инвестиционной деятельности очень важно. При этом особую важность имеет предварительный анализ, который проводится на стадии разработки инвестиционных проектов и способствует принятию разумных и обоснованных управленческих решений.

Весьма часто предприятие сталкивается с ситуацией, когда имеется ряд альтернативных (взаимоисключающих) инвестиционных проектов.

Естественно, возникает необходимость в сравнении этих проектов и выборе наиболее привлекательных из них по каким-либо критериям.

В инвестиционной деятельности существенное значение имеет фактор риска. Инвестирование всегда связано с иммобилизацией финансовых ресурсов предприятия и обычно осуществляется в условиях неопределенности, степень которой может значительно варьировать.

Результаты исследования. Исследование показывает что, обширная практика проведения реальных прогнозных расчетов инвестиционных проектов (ИП) свидетельствует о необходимости всестороннего учета различных видов неопределенности при оценке, планировании и управлении инвестиционными проектами. Действительность такова, что влияние факторов неопределенности на ИП приводит к возникновению непредвиденных 
ситуаций, приводящих к неожиданным потерям, убыткам, даже в тех проектах, которые первоначально признаны экономически целесообразными для предприятия, поскольку не учтенные в ИП негативные сценарии развития событий, пусть и малоожидаемые, тем не менее, могут произойти и сорвать реализацию инвестиционного проекта $[1,2]$. Учет неопределенности информации и его эффективность напрямую зависят от выбора математического аппарата, определяемого математической теорией. Этап обоснования и выбора математического аппарата, обеспечивающего приемлемую формализацию неопределенности и адекватное решение задач, возникающих при управлении реальными инвестициями, является крайне важным. Необоснованный и как, следствие, не правильный выбор математического аппарата, в основном, приводит к неадекватности созданных математических моделей, получению неверных результатов в процессе их применения и, соответственно, возникает недоверие к полученным результатам, и игнорируются выводы на их основе.

Выше проведенный анализ методов количественной оценки эффективности ИП в условиях неопределенности позволяет сделать вывод, что существующие методы, либо элиминируют неопределенность из модели ИП, что неправомерно, так как неопределенность является неотъемлемой характеристикой любого прогноза, либо неспособны формально описать, и учесть все возможное разнообразие видов неопределенности. Подавляющее большинство методов формализует неопределенности лишь в качестве распределений вероятностей, построенных на основе субъективных экспертных оценках, что в очень большом количестве случаев является явно идеализированным. Таким образом, в данных методах неопределенность, независимо от ее природы, отождествляется со случайностью [2], и поэтому они не позволяют учесть все возможное разнообразие видов неопределенностей, воздействующих на ИП. Как уже отмечалось, использование вероятностного подхода в инвестиционном анализе затрудняется причинами, связанными с отсутствием статистической информации или малым (недостаточным) размером выборки по некоторым из параметров ИП, что обусловлено уникальностью каждого ИП. Кроме того, точность оценки вероятностей (объективных и субъективных) зависит от множества факторов, начиная от качества статистической информации и заканчивая качеством экспертных оценок, поэтому и качество результирующей оценки эффективности и риска ИП слишком сильно зависит от них, что послужило росту недоверия к получаемым на их основе прогнозным оценкам и решениям. В связи с этим среди топ-менеджеров, банкиров, финансистов сложилось мнение, что подавляющее большинство прогнозных расчетов слишком идеализированы и далеки от практики. Многие предпочитают работать на основе опыта и интуиции. Это обусловлено, в том числе следующими основными причинами [3]:

- спецификой предметной области исследования, так как она находится на стыке современной прикладной математики, экономики и психологии;

- относительной новизной и недостаточной проработанностью математических методов анализа ИП в условиях неопределенности;

- низкой осведомленностью топ-менеджеров предприятий и специалистов в области финансов о новых математических подходах формализации и одновременной обработки разнородной информации (детерминированной, интервальной, лингвистической, статистической) и о возможностях построения на базе этих подходов специализированных методик.

Обширный опыт исследователей убедительно свидетельствует о том, что вероятностный подход не может быть признан надежным и адекватным инструментом решения слабоструктурированных задач [4], к которым принадлежат и задачи управления реальными инвестициями. В принципе, любая попытка использования статистических методов для решения такого рода задач есть не что иное, как редукция к хорошо структурированным (хорошо формализованным) задачам, при этом такого рода редукция существенно искажает исходную постановку задачи. Ограничения и недостатки применения «классических» формальных методов при решении слабоструктурированных задач являются следствием сформулированного основоположником теории нечетких множеств Л.А. Заде [5] «принципа несовместимости»: «...чем ближе мы подходим к решению проблем реального мира, тем очевиднее, что при увеличении сложности системы наша способность делать точные и уверенные заключения о ее поведении уменьшаются до определенного порога, за которым точность и уверенность становятся почти взаимоисключающими понятиями» [6].

Поэтому некоторыми исследователями разрабатываются методы оценки эффективности и риска инвестиционных проектов на основе аппарата теории нечетких множеств (ТНМ) [6]. В данных методах вместо распределения вероятности применяется распределение возможности, описываемое функцией принадлежности нечеткого числа.

Методы, базирующиеся на теории нечетких множеств, относятся к методам оценки и принятия решений в условия неопределенности. Их использование предполагает 
формализацию исходных параметров и целевых показателей эффективности ИП в виде вектора интервальных значений (нечеткого интервала), попадание в каждый интервал которого, характеризуется некоторой степенью неопределенности. Осуществляя арифметические и др. операции с такими нечеткими интервалами по правилам нечеткой математики, эксперты и лица принимаемых решений получают результирующий нечеткий интервал для целевого показателя. На основе исходной информации, опыта, и интуиции эксперты часто могут достаточно уверенно количественно охарактеризовать границы (интервалы) возможных (допустимых) значений параметров и области их наиболее возможных (предпочтительных) значений.

Также к методам, базирующихся на теории нечетких множеств, можно, в качестве частного случая, отнести давно и широко известный интервальный метод [4]. Данный метод соответствует ситуациям, когда достаточно точно известны лишь границы значений анализируемого параметра, в пределах которых он может изменяться, но при этом отсутствует какая-либо количественная или качественная информация о возможностях или вероятностях реализации различных его значений внутри заданного интервала. В соответствии с данным методом, входные переменные ИП задаются в виде интервалов, функции принадлежности которых, являются классическими характеристическими функциями множества, поэтому далее возможно прямое применение правил нечеткой математики для получения результирующего показателя эффективности ИП в интервальном виде. В интервальном методе за уровень (степень) риска предлагается принимать размер максимального ущерба, приходящегося на единицу неопределенности, т.е.:

$$
P=\frac{q_{N}-q_{\min }}{q_{\max }-q_{\min }}
$$

или

$$
P=\frac{q_{\max }-q_{N}}{q_{\max }-q_{\min }}
$$

где $q_{N}$ - требуемое значение параметра;

$q_{\min }$ - минимальное значение параметра;

$q_{\max }$ - максимальное значение параметра;

$P$ - уровень (степень) риска, или отношение расстояния от требуемой величины до ее минимального (максимального) значения к интервалу между ее максимальным и минимальным значениями.

Конкретный вариант выражения (1)-(2) зависит от используемого критерия эффективности. Например, для оценки риска ИП по критерию Чистая приведённая стоимость (ЧПС, чистая текущая стоимость, чистый дисконтированный доход, ЧДД, англ. Net present value, принятое в международной практике для анализа инвестиционных проектов сокращение - NPV) - это сумма дисконтированных значений потока платежей, приведённых к сегодняшнему дню необходимо использовать выражение (1), по критерию Direct Participation Program (DPP-nрограмма прямого участия) - (2). Такой способ определения риска полностью согласуется с геометрическим определением вероятности, однако при предположении, что все события внутри отрезка $\left[q_{\min }: q_{\max }\right]$ равновероятны. Очевидно, что данное предположение нельзя назвать отражающим реальную действительность.

При наличии дополнительной информации о значениях параметра внутри интервала, когда, например, известно, что значение $a$ более возможно, чем $b$, математическая формализация неопределенностей может быть адекватно реализована с помощью нечетко-интервального подхода. При использовании математического аппарата ТНМ экспертам необходимо формализовать свои представления о возможных значениях оцениваемого параметра ИП в терминах задания характеристической функции (функции принадлежности) множества значений, которые он может принимать. При этом от экспертов требуется указать множество тех значений, которые, по их мнению, оцениваемая величина не может принять (для них характеристическая функция равна 0), а затем, проранжировать множество возможных значений по степени возможности (принадлежности к данному нечеткому множеству). После того как формализация входных параметров инвестиционного проекта произведена, можно рассчитать распределение возможности $\mu_{\tilde{Y}}(y)$ выходного параметра (показателя эффективности ИП) $y$ по « $\alpha$-уровнему принципу обобщения» или «принципу обобщения Заде» [6]: 


$$
\mu_{\tilde{Y}}\left(y^{*}\right)=\sup _{\substack{f\left(x_{1}^{*}, x_{2}^{*}, \ldots, x_{n}^{*}\right)=y^{*} \\ x_{i}^{*} \in \operatorname{supp}\left(\tilde{X}_{i}\right), i=\overline{1, n}}}\left\{\min \left\{\mu_{\tilde{X}_{1}}\left(x_{1}^{*}\right), \mu_{\tilde{X}_{2}}\left(x_{2}^{*}\right), \ldots, \mu_{\tilde{X}_{n}}\left(x_{n}^{*}\right)\right\}\right\},
$$

где $\mu_{\tilde{X}_{i}}\left(x_{i}^{*}\right)$ - возможность того, что нечеткая величина $\tilde{X}_{i}$ примет значение $x_{i}^{*}$; $f\left(x_{1}^{*}, x_{2}^{*}, \ldots, x_{n}^{*}\right)=y^{*}-$ функциональная зависимость выходного параметра ИП от входных параметров.

Ниже перечислены основные преимущества нечетко-интервального подхода к оценке эффективности и риска инвестиционных проектов по сравнению с вышеперечисленными методами [4]:

1. Данный подход позволяет формализовать в единой форме и использовать всю доступную неоднородную информацию (детерминированную, интервальную, статистическую, лингвистическую), что повышает достоверность и качество принимаемых стратегических решений;

2. В отличие от интервального метода, нечетко-интервальный метод аналогично методу Монте-Карло, формирует полный спектр возможных сценариев развития ИП, а не только нижнюю и верхнюю границы, таким образом, инвестиционное решение принимается не на основе двух оценок эффективности ИП, а по всей совокупности оценок.

3. Нечетко-интервальный метод позволяет получить ожидаемую эффективность ИП как в виде точечного значения, так и в виде множества интервальных значений со своим распределением возможностей, характеризующимся функцией принадлежности соответствующего нечеткого числа, что позволяет оценить интегральную меру возможности получения отрицательных результатов от ИП, т.е. степень риска ИП.

4. Нечетко-интервальный метод не требует абсолютно точного задания функций принадлежности, так как в отличие от вероятностных методов, результат, получаемый на основе нечетко-интервального метода, характеризуется низкой чувствительностью (высокой робастностью (устойчивостью)) к изменению вида функций принадлежности исходных нечетких чисел, что в реальных условиях низкого качества исходной информации делает применение данного метода более привлекательным;

5. Вычисление оценок показателей ИП на основе нечетко-интервального метода оказывается эффективным в ситуациях, когда исходная информация, основана на малых статистических выборках, т.е. в случаях, когда вероятностные оценки не могут быть получены, что всегда имеет место при предварительной оценке долгосрочных инвестиций и достаточно часто - при последующем перспективном анализе, проводимом при отсутствии достаточной информационной базы;

6. Реализация нечетко-интервального метода на основе интервальной арифметики, предоставляет широкие возможности для применения данного метода в инвестиционном анализе, что обусловлено фактически отсутствием конкурентоспособных подходов к созданию надежного (в смысле гарантированности) и транспортабельности (по включению) инструментального средства для решения численных задач.

7. Характеризуется простотой выявления экспертных знаний.

Также нечетко-интервальный подход имеет преимущества в решении задач формирования оптимального портфеля инвестиционных проектов. Для решения задачи формирования оптимального портфеля ИП разработано большое количество моделей формирования оптимального портфеля ИП, отличающихся друг от друга видом целевых функций, свойствами переменных, используемыми математическими методами, учетом неопределенности. Как правило, для решения данной задачи используется аппарат линейного математического программирования в условиях определенности исходной информации: задача формулируется обычно как задача максимизации (или минимизации) заданной функции на заданном множестве допустимых альтернатив, которое описывается системой равенств или неравенств [6]. Например,

$$
f(x) \rightarrow \max , \text { при ограничениях } \phi_{i}(x) \leq 0, i=1, \ldots, m, x \in X,
$$

где $X$ - заданное множество альтернатив, $f: X \rightarrow R^{1}$ и $\phi: X \rightarrow R^{1}$ - заданные функции.

В качестве параметров целевой функции $f(x)$ для задачи формирования оптимального портфеля ИП используются различные интегральные показатели эффективности ИП, однако, несмотря на определенные преимущества и недостатки каждого из показателей, многие исследователи склоняются к тому, что наиболее предпочтительным представляется использование $N P V$ в качестве параметров целевой функции $[5,6]$, прежде всего потому, что $N P V$ обладает свойством аддитивности, что дает возможность оценить доходность всего 
портфеля ИП как сумму доходностей отдельных ИП, образующих данный портфель. Возможны различные варианты постановки задачи формирования оптимального портфеля ИП. Чаще всего, экономический смысл целевой функции $f(x)$ состоит в максимизации экономического эффекта от инвестиционной деятельности, а смысл ограничений $\phi_{i}(x) \leq 0$, налагаемых на множество допустимых решений задачи, отражает ограниченность денежных средств с учетом возможности различных бюджетных ограничений для каждого из временных отрезков действия проекта.

Так как стратегические решения, в том числе связанные с формированием оптимального портфеля инвестиционных проектов, направлены на долгосрочную перспективу и, следовательно, по своей природе сопряжены со значительной неопределенностью, а также имеют значительную субъективную составляющую, поэтому применение нечеткого математического программирования к решению задачи формирования оптимального портфеля ИП обладает многими преимуществами.

В качестве примера можно рассмотреть ситуацию, в которой множество допустимых альтернатив (инвестиционных проектов) представляет собой совокупность всевозможных способов распределения ресурсов, которые ЛПР собирается вложить с целью формирования оптимального инвестиционного портфеля. Очевидно, что, в этом случае, нецелесообразно заранее вводить четкую границу для множества допустимых альтернатив (например, четких ограничений на размер инвестиционного бюджета предприятия в период $t$ ), поскольку может случиться так, что распределения ресурсов (инвестиционные проекты), незначительно лежащие за этой границей (т.е. вне ограничений), дадут эффект, «перевешивающий» меньшую желательность (например, по размеру инвестиционных затрат) этих распределений для ЛПР. Таким образом, нечеткое описание оказывается более адекватным реальности, чем в определенном смысле произвольно принятое четкое описание задачи.

Выводы. Таким образом, сравнительный анализ традиционных методов оценки эффективности долгосрочных инвестиций, существующих методов формирования оптимального портфеля ИП и нечетко-интервального метода показал, что ТНМ является одной из наиболее эффективных математических теорий, направленных на формализацию и обработку неопределенной информации и во многом интегрирующей известные подходы и методы. ТНМ в очередной раз подтверждает широко известную исследователям истину: применяемый формальный аппарат по своим потенциальным возможностям и точности должен быть адекватен семантике, и соответствовать точности используемых исходных данных. Поэтому методы математического анализа эффективно применяются при точных исходных данных. Математическая статистика и теория вероятностей используют экспериментальные данные, обладающие строго определенной точностью и достоверностью. Теория нечетких множеств позволяет обрабатывать разнородную информацию, характерную для реальных задач инвестиционного анализа.

\section{ЛИТЕРАТУРА}

1. Алтунин А. Е., Семухин М. В. Модели и алгоритмы принятия решений в нечетких условиях. - Тюмень: Изд-во ТГУ, 2012. - 352 с.

2. Бирман Г., Шмидт С. Экономический анализ инвестиционных проектов. - М.: ЮНИТИ, 2010. - 345 с.

3. Борисов А. Н., Алексеев А. В., Меркурьева Г. В. и др. Обработка нечеткой информации в системах принятия решений. - М: Радио и связь. 1989. - 304 с.

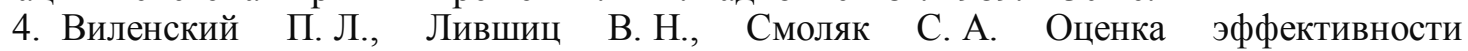
инвестиционных проектов. Теория и практика. - М.: Дело, 2011. - 888 с.

5. Заде Л. А. Основы нового подхода к анализу сложных систем и процессов принятия решений.- В кн.: Математика сегодня. - М.: Знание, 1974, с. 5-49.

6. Заде Л. Понятие лингвистической переменной и его применение к принятию приближенных решений: Пер. с англ. - М.: Мир, 1976. - 165 с. 